\title{
ChemComm
}

\section{Coherent electron spin manipulation in a dilute oriented ensemble of molecular nanomagnets: pulsed EPR on doped single crystals $\dagger$}

Cite this: Chem. Commun., 2014 50, 91

Received 17th August 2013 Accepted 29th October 2013

DOI: $10.1039 / c 3 c c 46326 e$

www.rsc.org/chemcomm
Fabrizio Moro, ${ }^{a}$ Danielle Kaminski, ${ }^{b}$ Floriana Tuna, ${ }^{a}$ George F. S. Whitehead, ${ }^{a}$ Grigore A. Timco, ${ }^{a}$ David Collison, ${ }^{a}$ Richard E. P. Winpenny, ${ }^{a}$ Arzhang Ardavan ${ }^{b}$ and Eric J. L. Mclnnes*a
Doping a $\mathrm{Cr}_{7} \mathrm{Zn}$ molecular nanomagnet into a diamagnetic and isostructural host allows pulsed X-band EPR on single crystals, including relaxation and nutation experiments on the $S=3 / 2$ ground state.

The study of coherent electron spin dynamics in molecular nanomagnets (MNMs) has recently become the subject of intense research. ${ }^{1-9}$ This is inspired in part by proposals for quantum information processing, ${ }^{10}$ exploiting, for example, the large total spins and molecular size, intra-molecular excitations, and the ability to make more complex structures via supramolecular approaches. ${ }^{11}$ It is also important generally in the study of how such molecules interact with their environment. ${ }^{8}$ Pulsed EPR allows direct measurement of spin coherence; the first such studies for MNMs, on $\mathrm{Cr}_{7} \mathrm{M}$ heterometallic rings, revealed phase memory times $\left(T_{\mathrm{M}}\right.$; quantifying the rate of loss of quantum phase information to the environment) of the order of $\mu \mathrm{s}$ at a few kelvin. ${ }^{1}$ This and subsequent ${ }^{2-6}$ studies were performed on dilute solutions, or doped into nanoporous silicon, ${ }^{5}$ in order to minimise decoherence due to intermolecular dipolar interactions. However, the sample is then an ensemble of randomly oriented molecules. Various proposals for MNMs depend on coherent manipulation of an anisotropic spin multiplet. ${ }^{10}$ Since transition energies depend on orientation, the lack of alignment limits the range of quantum information experiments that may be performed.

The obvious way to address aligned samples is in single crystals. This requires alternative strategies to overcome inter-molecular driven decoherence. Takahashi et al. reported pulsed $240 \mathrm{GHz}$ EPR on crystals of $\mathrm{Fe}_{8}$ under very high magnetic fields that quench electron spin flip-flop relaxation. ${ }^{7,8}$ The alternative is to dilute the MNM in an isostructural and diamagnetic host. This is straightforward for monometallic complexes, ${ }^{9}$ but for high nuclearity d-block

\footnotetext{
${ }^{a}$ School of Chemistry and Photon Science Institute, The University of Manchester, Oxford Road, Manchester M13 9PL, UK. E-mail: eric.mcinnes@manchester.ac.uk; Fax: +44 (0)161-275-1001; Tel: +44 (0)161-275-4469

${ }^{b}$ CAESR, The Clarendon Laboratory, Department of Physics, University of Oxford, Parks Road, Oxford OX1 3PU, UK. E-mail: a.ardavan1@physics.ox.ac.uk;

Tel: +44 (0)1865 272366

$\dagger$ Electronic supplementary information (ESI) available: Syntheses; unit cell details; EPR spectra, crystal mounting, relaxation and nutation data. CCDC 954609 and 954610. For ESI and crystallographic data in CIF or other electronic format see DOI: 10.1039/c3cc46326e
}

clusters it is rarely possible to prepare the diamagnetic equivalent. Here we present single-crystal pulsed X-band EPR studies on $\left(\mathrm{Me}_{2} \mathrm{NH}_{2}\right)\left[\mathrm{Cr}_{7} \mathrm{ZnF}_{8}\left(\mathrm{O}_{2} \mathrm{C}^{t} \mathrm{Bu}\right)_{16}\right]\left(\mathrm{Cr}_{7} \mathrm{Zn}\right)$, achieved by doping into the diamagnetic $\mathrm{Ga}_{7} \mathrm{Zn}$ analogue.

$\mathrm{Cr}_{7} \mathrm{Zn}$ and $\mathrm{Ga}_{7} \mathrm{Zn}$ were prepared by adaptations of methods for other $\mathrm{M}^{\prime}{ }_{7} \mathrm{M}(\mathrm{ESI} \dagger) .{ }^{12}$ The metal ions in each molecule define a planar octagon, with each edge bridged by a fluoride and two pivalates, giving a complex anion which is centred on a $\mathrm{Me}_{2} \mathrm{NH}_{2}^{+}$cation (Fig. S1, ESI ${ }^{\dagger}$ ). Single crystals of $\mathrm{Cr}_{7} \mathrm{Zn}$ (0.3\% by mass) doped in $\mathrm{Ga}_{7} \mathrm{Zn}\left(\mathrm{Cr}_{7} \mathrm{Zn}\right.$ @ $\mathrm{Ga} Z \mathrm{Zn}$ ), isostructural with the parent compounds, were grown from toluene. CW EPR (Fig. S2, ESI†) shows the Cr7Zn dopant is incorporated without scrambling of $\mathrm{Cr}^{\mathrm{III}}$ and $\mathrm{Ga}^{\mathrm{III}}$. $\mathrm{Cr}_{7} \mathrm{Zn}$ has a well defined total spin $S=3 / 2$ ground state, as characterised by $\mathrm{INS}^{13}$ and CW EPR, ${ }^{14}$ because the hetero-ion prevents complete cancellation of spin despite the antiferromagnetic coupling. At $5 \mathrm{~K}$ over $90 \%$ of the population (>99\% at $2 \mathrm{~K}$ ) is in the ground state, which therefore dominates the EPR, with the remainder in the $S=1 / 2$ and 5/2 lowest excited states which lie at 6.7 and $15 \mathrm{~cm}^{-1}$, respectively, in zero-field. ${ }^{14}$ The ground state has axial and rhombic zero-field splitting (ZFS) parameters of $D=-0.4186$ and $E=-0.0489 \mathrm{~cm}^{-1}$, respectively [spin Hamiltonian (1) with $g_{\text {iso }}=1.96 ; \mathbf{B}$ is the applied magnetic field]. The ZFS is largely dominated by the single ions, but with important contributions from anisotropic exchange. ${ }^{14}$ The principal direction of the $\mathbf{D}$ tensor (the molecular $z$ axis) is normal to the $\mathrm{Cr}_{7} \mathrm{Zn}$ plane (the $x y$ plane).

$$
\hat{H}_{\mathrm{s}}=\mu_{\mathrm{B}} \mathbf{B} g \hat{S}+D\left[\hat{S}_{z}^{2}-S(S+1) / 3\right]+E\left(\hat{S}_{x}^{2}-\hat{S}_{y}^{2}\right)
$$

$\mathrm{Cr}_{7} \mathrm{Zn} @ \mathrm{Ga}_{7} \mathrm{Zn}$ crystallises in the tetragonal $I_{4}$ space group (ESI + ), with the molecules lying on the $C_{4}$ axis such that the $\mathrm{M}^{\prime}{ }_{7} \mathrm{Zn}$ rings are in the crystal $a b$ plane (Fig. 1). Crystals can be conveniently mounted (Fig. S3, ESI $\dagger$ ) to allow EPR measurement in the $b c$ plane (Fig. 2). The $b$ and $c$ axes (which we define as $\theta=90$ and $0^{\circ}$, respectively) are readily identified from the turning points in the resonance field road map (Fig. S4, ESI $\dagger$ ). The coplanarity of the rings in the unit cell means there is only one magnetically distinct molecule when $\mathbf{B}$ is parallel to $c$ (hence the molecular $z$ axis). However, because the $\mathrm{Zn}$ ion is disordered over all sites of the $\mathrm{Cr}_{7} \mathrm{Zn}$ octagon, when $\mathbf{B}$ is off the $c$ axis there are magnetically distinct molecules: if $\varphi$ is the projection of $\mathbf{B}$ onto the molecular $x y$ plane then these correspond to $\varphi, \varphi+45^{\circ}, \varphi+90^{\circ}$ and 


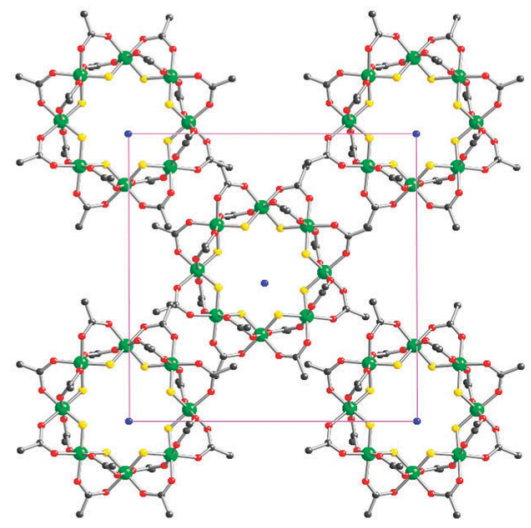

Fig. 1 Unit cell of $\mathrm{Cr}_{7} Z \mathrm{ZnaGa} Z \mathrm{Zn}$ viewed down the crystal $c$ axis: $\mathrm{Ga} / \mathrm{Cr} / \mathrm{Zn}$ (green), F (yellow), O (red), N (blue), C (black), Me groups omitted for clarity. See Fig. S1 (ESI†) for full molecule.

$\varphi+135^{\circ}$ (further rotations about $c$ are equivalent). Hence we should observe four sets of resonances from distinct molecules in the $b c$ plane, collapsing to one set at $c$. We have calculated ${ }^{16}$ the single orientation spectra with this model, using the $S=3 / 2$ parameters above; i.e. with no free variables other than a molecular azimuthal angle $\varphi$ which defines all four magnetically distinct molecules by symmetry. Good agreement is found for $\varphi=16^{\circ}$ (Fig. 2 and Fig. S4, ESI $\dagger$ ). The simulations use a natural linewidth of $4 \mathrm{mT}$ with a $D$-strain of $15 \%$ and a crystal mosaicity ${ }^{15}$ of $c a .2 \%$.

The splitting of the spectrum into four inequivalent sites is most clearly resolved in the low-field transitions (Fig. 2), which have much narrower linewidths. This latter phenomenon is a result of the states involved in the transitions, as shown by calculated Zeeman diagrams for the $S=3 / 2$ multiplet with B orientated just off the $c$ axis and along $b$ (Fig. 3). The low-field transitions are within a single Kramers doublet, while the high-field transitions are between the mixed $| \pm 1 / 2\rangle$ and $| \pm 3 / 2\rangle$ doublets. Hence, the former transitions are

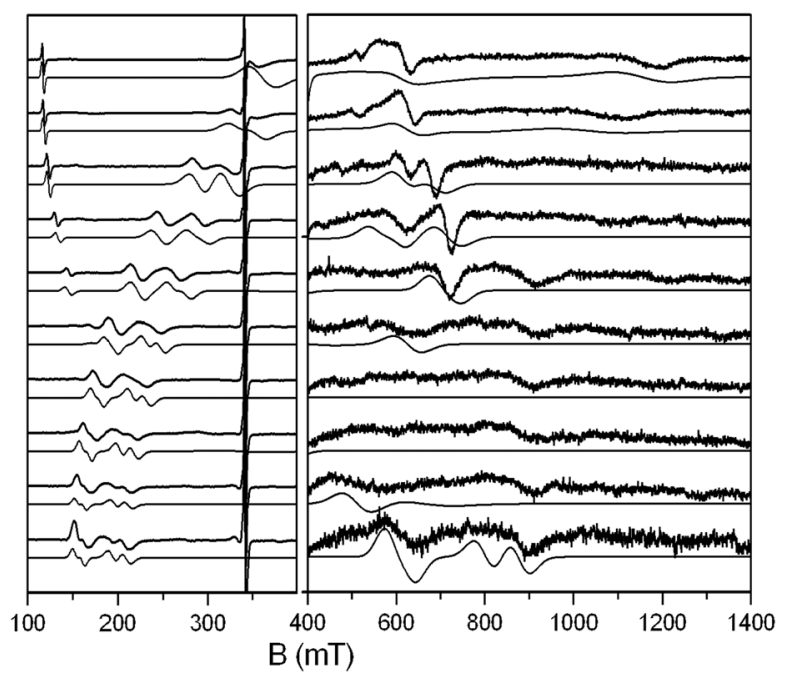

Fig. 2 Single crystal CW X-band EPR of $\mathrm{Cr}_{7} Z n$ a Ga $a_{7} Z n$ in the bc plane at $5 \mathrm{~K}$ : experimental (thick) and simulated spectra (thin; summation over four molecular orientations, see text). Crystal orientations from $\theta=0$ (top) to $90^{\circ}$ (bottom); high-field regions $\times 20$ for clarity. [The sharp feature at $g=$ $1.96(342 \mathrm{mT})$ is due to the $S=1 / 2$ excited state.]
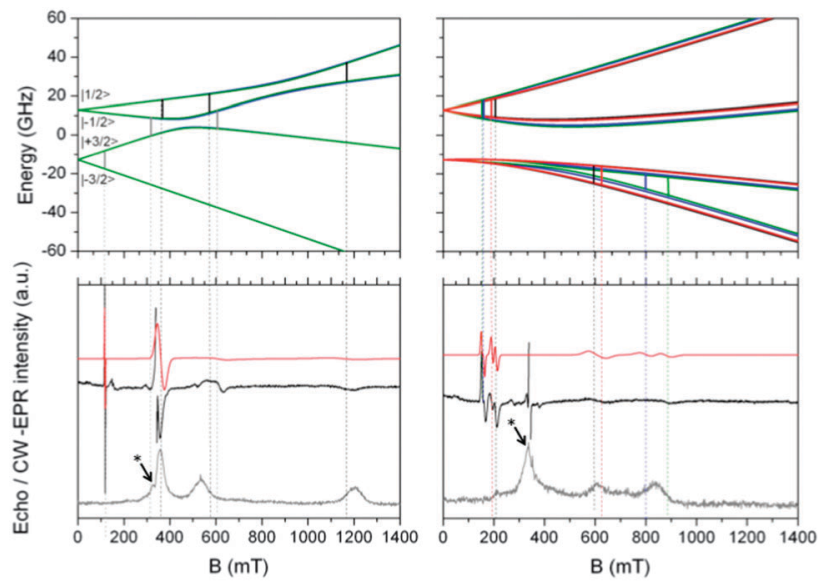

Fig. 3 Top: Zeeman diagrams for the $S=3 / 2$ ground state and $X$-band EPR transitions calculated for $\theta=8^{\circ}$ (left) and $90^{\circ}$ (right; different colours represent the four molecular orientations, see text). Bottom: experimental (black) and simulated (red) CW, and FSED (grey) spectra at $5 \mathrm{~K}$. [The peak at $330 \mathrm{mT}$, marked *, is intrinsic to the resonator.]

independent of the ZFS and are unaffected by $D$-strain. ${ }^{14}$ In contrast the latter transitions are significantly broadened by strain effects.

The doping reduces intermolecular effects sufficiently to allow single crystal pulsed EPR: we observe spin echoes with a standard Hahn sequence $(\pi / 2-\tau-\pi-\tau$-echo) at temperatures below $c a .8 \mathrm{~K}$. Xband field-swept echo-detected (FSED) spectra were measured on a polycrystalline sample (Fig. S5, ESI $\dagger$ ) and on a single crystal at $\theta=0$ and $90^{\circ}$ (Fig. 3 and Fig. S5, ESI $\dagger$ ) by integration of the echo intensity at various static applied fields (16 ns $\pi / 2$ pulse with fixed delay $\tau=$ $200 \mathrm{~ns})$. There is a clear correspondence of the $S=3 / 2$ ground state resonances between the CW and FSED spectra.

$T_{\mathrm{M}}$ was measured for the 366,545 and $1220 \mathrm{mT}$ transitions at $c \| \mathbf{B}$ by monitoring the decay of the echo intensity as a function of $\tau$ and fitting to a stretched exponential function (Fig. S6, ESI $†$; ESEEM modulations due to ${ }^{1} \mathrm{H}$ are observed). $T_{\mathrm{M}}$ increases gradually from 5 to $2.1 \mathrm{~K}$, reaching $0.87,0.66$ and $0.58 \pm 0.01 \mu$ s respectively (Fig. S7, ESI†). It should be possible to significantly enhance these values by: (i) further dilution. A $0.3 \%$ dopant level gives a statistical range of inter-spin separations and consequent dipolar interactions centred round $c a .500 \mathrm{kHz}$. This frequency would put an upper limit of ca. $2 \mu \mathrm{s}$ on the spin-spin relaxation time $T_{2}$, comparable with our $T_{\mathrm{M}}$; (ii) per-deuteration, reducing dipolar interactions with nuclear spins; ${ }^{1}$ (iii) using carboxylates and cation without methyl groups, reducing spectral diffusion effects due to Me rotations and librations. We have previously achieved $c a$. ten-fold enhancements of $T_{\mathrm{M}}$ for $\mathrm{Cr}_{7} \mathrm{Ni}$ in dilute solution by these methods. ${ }^{6}$ The $T_{\mathrm{M}}$ values observed here are similar to those measured for $\mathrm{Cr}_{7} \mathrm{Ni}$ with the same ligand set; ${ }^{6}$ this has an $S=1 / 2$ ground state hence phonon driven modulation of the ZFS does not appear to be a dominant relaxation mechanism. $T_{\mathrm{M}}$ values measured for $b \| \mathbf{B}$ at $4.5 \mathrm{~K}$ are in the range of those measured for $c \| \mathbf{B}\left(0.56\right.$ and $0.45 \mu \mathrm{s}$ for $B_{0}=612$ and $\left.840 \mathrm{mT}\right)$, consistent with observations on $\mathrm{Cr}_{7} \mathrm{Mn}(S=1)$ in frozen solution that $T_{\mathrm{M}}$ varies little at different points (orientations) in the spectrum. ${ }^{1}$

Finally we show that we can perform coherent spin manipulations on the $\mathrm{Cr}_{7} \mathrm{Zn} @ \mathrm{Ga}_{7} \mathrm{Zn}$ single crystal by nutation experiments. In this experiment, a nutation pulse of duration $t_{\mathrm{p}}$ tips the 


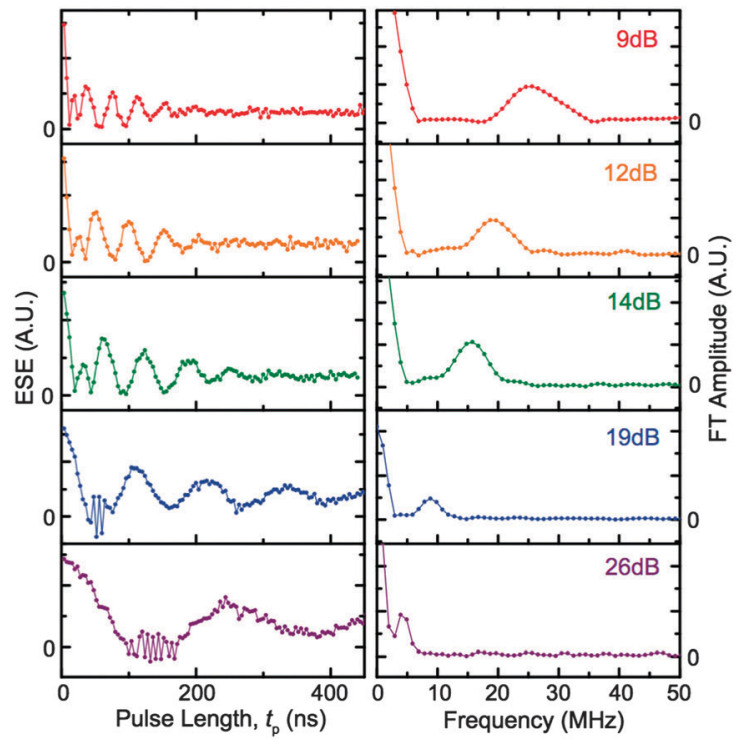

Fig. 4 Left: nutation experiment at $4.5 \mathrm{~K}$ measured at $B_{0}=1160 \mathrm{mT}$ with $c \| \mathbf{B}$, for a range of attenuation settings. Right: Fourier transforms of the nutations.

magnetisation through an angle $g \mu_{\mathrm{B}} B_{1} t_{\mathrm{p}} / \hbar$, followed by a two-pulse detection sequence $\left[t_{\mathrm{p}}-T-\pi / 2-\tau-\pi-\tau\right.$-echo, with fixed $T$ and 4 -step phase cycling]. The echo intensity, quantifying the $z$-component of the magnetisation after the nutation pulse, is measured as function of $t_{\mathrm{p}}$. This experiment sets up a coherent oscillation between the two states, corresponding to generation of arbitrary superpositions, observed as oscillation of the echo intensity as a function of $t_{\mathrm{p}}$. Some authors describe these as Rabi oscillations, although this is only strictly correct when the pulse bandwidth substantially exceeds the EPR linewidth such that all spin packets rotate together.

We selected the highest-field and most isolated transition for $c \| \mathbf{B}$, with $B_{0}=1160 \mathrm{mT}$, corresponding to the nominally $m_{\mathrm{s}}=+1 / 2$ to $+3 / 2$ transition with frequency $9.77 \mathrm{GHz}$. We find oscillations in the echo amplitude as a function of $t_{\mathrm{p}}$ (Fig. $4 ; T=256 \mathrm{~ns}$ and the $\pi / 2$ - and $\pi$-pulses are optimised for the power). The oscillation frequency is proportional to the square root of microwave power (i.e. to the amplitude of the microwave field $B_{1}$; Fig. S8, ESI $\dagger$ ), demonstrating that we are observing coherent electron spin oscillations rather than other phenomena such as ESEEM. The excitation bandwidth in this experiment is narrow compared to the inhomogeneous broadening of the line, leading to a nutation envelope that depends on oscillation number rather than time.

It has been noted in previous nutation experiments on MNMs in frozen solutions ${ }^{2-4}$ that the distribution in molecular orientations being excited (each with a different nutation frequency) contributes to premature damping of the oscillations before the limits placed by $T_{\mathrm{M}}$. We have removed this factor in this single crystal experiment and the limiting factors may include $D$-strain and/or crystal mosaicity, with analogous effects.

In conclusion, we have reported the first pulsed EPR on single crystals of doped MNMs. This opens the way for coherent multifrequency experiments manipulating the quantum spin state, thereby testing proposals for their application in quantum information processing. This work highlights the utility of the $\mathrm{M}_{7}{ }_{7} \mathrm{M}$ family in testing new theories and experiments for MNMs, due to their tuneable composition and magnetic properties. ${ }^{16}$ In this case, doping without scrambling of the metal ions is aided by the kinetic inertness of the $\mathrm{Cr}$ (III) ion. However, the fact that an $\mathrm{Fe}_{4}$-doped $\mathrm{Ga}_{4}$ material has been synthesised (though pulsed EPR on it was not reported), ${ }^{17}$ indicates that it is possible with more labile metal ions. Future targets will also include doped dimers of $S=1 / 2$ ground state MNMs, which we can prepare with tuneable interaction strengths, ${ }^{18}$ for potential use in logic gates in analogy to the organic-based chemistry of Takui and co-workers on doped single crystals of nitroxyl dimers. ${ }^{19}$

We are grateful to the EPSRC for funding, including for the National EPR Facility at Manchester and the Oxford CAESR. REPW thanks the Royal Society for a Wolfson Merit Award.

\section{Notes and references}

1 A. Ardavan, O. Rival, J. J. L. Morton, S. J. Blundell, A. M. Tyryshkin, G. A. Timco and R. E. P. Winpenny, Phys. Rev. Lett., 2007, 98, 057201.

2 G. Mitrikas, Y. Sanakis, C. P. Raptopoulou, G. Kordas and G. Papavassiliou, Phys. Chem. Chem. Phys., 2008, 10, 743.

3 C. Schlegel, J. van Slageren, M. Manoli, E. K. Brechin and M. Dressel, Phys. Rev. Lett., 2008, 101, 147203.

4 S. Bertaina, S. Gambarelli, T. Mitra, B. Tsukerblat, A. Müller and B. Barbara, Nature, 2008, 453, 203.

5 K.Y. Choi, Z. Wang, H. Nojiri, J. van Tol, P. Kumar, P. Lemmens, B. S. Bassil, U. Kortz and N. S. Dalal, Phys. Rev. Lett., 2012, 108, 067206.

6 C. J. Wedge, G. A. Timco, E. T. Spielberg, R. E. George, F. Tuna, S. Rigby, E. J. L. McInnes, R. E. P. Winpenny, S. J. Blundell and A. Ardavan, Phys. Rev. Lett., 2012, 108, 107204.

7 S. Takahashi, J. van Tol, C. C. Beedle, D. N. Hendrickson, L.-C. Brunel and M. S. Sherwin, Phys. Rev. Lett., 2009, 102, 087603.

8 S. Takahashi, I. S. Tupitsyn, J. van Tol, D. N. Hendrickson and P. C. E. Stamp, Nature, 2011, 476, 76.

9 M. J. Martinez-Perez, S. Serra-Cardona, C. Schlegel, F. Moro, P. J. Alonso, H. Prima-Garcia, J. M. Clemente-Juan, M. Evangelisti, A. Gaita-Arino, J. van Slageren, E. Coronado and F. Luis, Phys. Rev. Lett., 2012, 108, 247213.

10 For example: M. N. Leuenberger and D. Loss, Nature, 2001, 410, 789; F. Meier, J. Levy and D. Loss, Phys. Rev. Lett., 2003, 90, 047901; F. Troiani, M. Affronte, P. Santini, S. Carretta and G. Amoretti, Phys. Rev. Lett., 2005, 94, 190501.

11 S. Hill, R. S. Edwards, N. Aliaga-Alcade and G. Christou, Science, 2003, 302, 1015; G. A. Timco, E. J. L. McInnes, R. G. Pritchard, F. Tuna and R. E. P. Winpenny, Angew. Chem., Int. Ed., 2008, 47, 9681.

12 F. K. Larsen, J. Overgaard, S. Parsons, E. Rentschler, A. A. Smith, G. A. Timco and R. E. P. Winpenny, Angew. Chem., Int. Ed., 2003, 42, 5978; E. C. Sañudo, C. A. Muryn, M. A. Helliwell, G. A. Timco, W. Wernsdorfer and R. E. P. Winpenny, Chem. Commun., 2007, 801.

13 R. Caciuffo, T. Guidi, G. Amoretti, S. Carretta, E. Liviotti, P. Santini, C. Mondelli, G. Timco, C. A. Muryn and R. E. P. Winpenny, Phys. Rev. B, 2005, 71, 174407.

14 S. Piligkos, E. Bill, D. Collison, E. J. L. McInnes, G. A. Timco, H. Weihe, R. E. P. Winpenny and F. Neese, J. Am. Chem. Soc., 2007, 129, 760; S. Piligkos, H. Weihe, E. Bill, F. Neese, H. El Mkami, G. M. Smith, D. Collison, G. Rajaraman, G. A. Timco, R. E. P. Winpenny and E. J. L. McInnes, Chem.-Eur. J., 2009, 15, 3152.

15 Using Weihe's program SimEPR, see: C. J. Jacobsen, E. Pederson, J. Villadsen and H. Weihe, Inorg. Chem., 1993, 32, 1216; S. Klitgaard, F. Glasbol and H. Weihe, Spectrochim. Acta, Part A, 2006, 63, 836.

16 R. H. Laye, F. K. Larsen, J. Overgaard, C. A. Muryn, E. J. L. McInnes, E. Rentschler, V. Sanchez, S. J. Teat, H. U. Güdel, O. Waldmann, G. A. Timco and R. E. P. Winpenny, Chem. Commun., 2005, 1125; G. A. Timco, E. J. L. McInnes and R. E. P. Winpenny, Chem. Soc. Rev., 2013, 42, 1796.

17 L. Vergnani, A.-L. Barra, P. Neugebauer, M. J. Rodriguez-Douton, R. Sessoli, L. Sorace, W. Wernsdorfer and A. Cornia, Chem.-Eur. J., 2012, 18, 3390.

18 T. B. Faust, V. Bellini, A. Candini, S. Carretta, G. Lorusso, D. R. Allan, L. Carthy, D. Collison, R. J. Docherty, J. Kenyon, J. Machin, E. J. L. McInnes, C. A. Muryn, H. Nowell, R. G. Pritchard, S. J. Teat, G. A. Timco, F. Tuna, G. F. S. Whitehead, W. Wernsdorfer, M. Affronte and R. E. P. Winpenny, Chem.-Eur. J., 2011, 17, 14020.

19 S. Nakazawa, S. Nishida, T. Ise, T. Yoshino, N. Mori, R. D. Rahimi, K. Sato, Y. Morita, K. Toyota, D. Shiomi, M. Kitagawa, H. Hara, P. Carl, P. Höfer and T. Takui, Angew. Chem., Int. Ed., 2012, 51, 9860. 